\title{
3D imaging of the pion off-shell electromagnetic form factors *
}

\author{
Ho-Meoyng Choi ${ }^{\dagger}$ \\ Department of Physics Education, Kyungpook National University, Daegu 41566, Korea \\ E-mail: homyoungeknu.ac.edu \\ T. Frederico \\ Instituto Tecnológico de Aeronáutica, 12.228-900 São José dos Campos, SP, Brazil \\ E-mail: tobiaseita.br
}

\section{Chueng-Ryong Ji}

Department of Physics, North Carolina State University, Raleigh, North Carolina 27695-8202, USA

E-mail: crji@ncsu.edu

\section{J. P. B. C. de Melo}

Laboratório de Física Teórica e Computacional, Universidade Cruzeiro do Sul/Universidade Cidade de São Paulo, 01506-000, São Paulo SP, Brazil

E-mail: joao.mello@cruzeirodosul.edu.br

\begin{abstract}
We study the pion electromagnetic half-off-shell form factors $F_{1}$ and $F_{2}$ using an exactly solvable manifestly covariant model of a $(3+1)$ dimensional fermion field theory. The model provides a three-dimensional imaging of $F_{1}$ and $F_{2}$ as a function of $\left(Q^{2}, t\right)$, which are constrained by the Ward-Takahashi identity. The normalization of the charge form factor $F_{1}$ is fixed by $F_{1}\left(Q^{2}=\right.$ $\left.0, t=m_{\pi}^{2}\right)=1$ while the other form factor $F_{2}$ vanishes, i.e. $F_{2}\left(Q^{2}, t=m_{\pi}^{2}\right)=0$ for any value of $Q^{2}$ due to the time-reversal invariance of the strong interaction. The new form factor defined by $g\left(Q^{2}, t\right)=F_{2}\left(Q^{2}, t\right) /\left(t-m_{\pi}^{2}\right)$ is however measurable in the on-mass-shell limit. We note that $g\left(Q^{2}=0, t=m_{\pi}^{2}\right)$ is related with the pion charge radius.
\end{abstract}

Light Cone 2019 - QCD on the light cone: from hadrons to heavy ions - LC2019

16-20 September 2019

Ecole Polytechnique, Palaiseau, France

\footnotetext{
*This work was supported in part by the US Department of Energy under Grant No. DE-FG02-03ER41260 (CJ), by the National Research Foundation of Korea (NRF) under Grant No. NRF-2017R1D1A1B03033129 (HMC), by the project INCT-FNA Proc. No. 464898/2014-5, by CAPES - Finance Code 001, by Conselho Nacional de Desenvolvimento Científico e Tecnológico (CNPq) under grants 308025/2015-6 (JPBCM), 308486/2015-3 (TF), PVE 401322/2014-9 (CJ), by Fundação de Amparo à Pesquisa do Estado de São Paulo (FAPESP) under the thematic projects 2013/26258-4 and 2017/05660-0, and by regular project 2019/02923-5 (JPBCM).

${ }^{\dagger}$ Speaker.
} 


\section{Introduction}

The pion is the simplest hadronic system, the valence structure of which is a bound state of a quark and an antiquark. Its structure is known to be parametrized by a single on-mass-shell electromagnetic (EM) form factor, $F_{\pi}\left(Q^{2}\right)$, which depends on the 4-momentum squared $q^{2}\left(=-Q^{2}\right)$ of the virtual photon. While $F_{\pi}\left(Q^{2}\right)$ for the low spacelike momentum transfers $Q^{2}$ has been measured directly by elastic scattering of high-energy mesons off atomic electrons, the extraction of $F_{\pi}\left(Q^{2}\right)$ to higher $Q^{2}$ regions through elastic scattering is very difficult experimentally. Thus, $F_{\pi}\left(Q^{2}\right)$ for the higher $Q^{2}$ values has been extracted from the pion electroproduction reaction by exploiting the nucleon's pion cloud as a target, i.e., $F_{\pi}\left(Q^{2}\right)$ has been extracted from the measurements of the cross sections for the reaction ${ }^{1} \mathrm{H}\left(e, e^{\prime} \pi^{+}\right) n$ up to values of $Q^{2}=3.91 \mathrm{GeV}^{2}[1,2,3]$. However, the main problem in using the electroproduction process as a tool for accessing a "pion target" is that the pions in a nucleon's cloud are not real but virtual particles. Accordingly, one cannot access the form factor at the exact pion pole in the actual experiment as the extrapolation to $t \rightarrow m_{\pi}^{2}$ involves the disallowed kinematic region of the electroproduction $(t<0)$. This may raise some questions about the validity of the extrapolation from the off-shell results to the on-shell limit. Furthermore, the EM structure of the off-shell hadron is more complicated than the on-shell hadron and involves more form factors [4]. For instance, the off-shell EM structure of the pseudoscalar meson [4] requires two form factors, which are related by the Ward-Takahashi identity(WTI).

In this work, we discuss the electromagnetic off-shell effects for the pion using an exactly solvable manifestly covariant model of $(3+1)$ dimensional fermion field theory and compare the two off-shell form factors $F_{1}\left(Q^{2}, t\right)$ and $F_{2}\left(Q^{2}, t\right)$ with the data extracted from the pion electroproduction reaction [1]. More detailed analysis can be found in Ref. [5].

\section{Off-shell Pion Electromagnetic Form Factors}

The most general parametrization of the vertex function $\Gamma^{\mu}$ for the half-on-shell $\left(p^{2}=m_{\pi}^{2}\right)$ and half-off-shell $\left(p^{2}=t<0\right)$ electromagnetic form factors of the charged pion can be given in terms of the initial and final 4-momenta, $p^{\mu}$ and $p^{\prime \mu}\left(=p^{\mu}+q^{\mu}\right)$, as

$$
\Gamma_{\mu}=\left(p^{\prime}+p\right)_{\mu} F_{1}\left(Q^{2}, t\right)+q_{\mu} F_{2}\left(Q^{2}, t\right) .
$$

where $F_{2}\left(Q^{2}, t\right)=\left(t-m_{\pi}^{2}\right)\left[F_{1}(0, t)-F_{1}\left(Q^{2}, t\right)\right] / Q^{2}$ is obtained from the WTI [5]. It is known that $F_{2}\left(Q^{2}, t\right)$ cannot be directly measured in the electroproduction process due to the transversality of the electron current. We note, however, that the new form factor $g\left(Q^{2}, t\right) \equiv F_{2}\left(Q^{2}, t\right) /\left(t-m_{\pi}^{2}\right)$ is nonzero in the limit of $t \rightarrow m_{\pi}^{2}$ although $F_{2}\left(Q^{2}, t\right)$ itself goes to zero as $t \rightarrow m_{\pi}^{2}$. The form factor $g\left(Q^{2}, m_{\pi}^{2}\right)$ is the new observable in the on-mass-shell limit besides the usual charge form factor $F_{1}\left(Q^{2}, m_{\pi}^{2}\right)$ and should be measurable in the experiment of pion electroproduction. The off-shell form factor $F_{2}\left(Q^{2}, t\right)$ can be rewritten as the following sum rule,

$$
F_{1}\left(Q^{2}, t\right)-F_{1}(0, t)+Q^{2} g\left(Q^{2}, t\right)=0 .
$$

Especially, taking the derivative of this sum rule, we find [5] in the on-mass-shell limit $t=m_{\pi}^{2}$ and $Q^{2}=0$ that $g\left(Q^{2}=0, m_{\pi}^{2}\right)=\left\langle r_{\pi}^{2}\right\rangle / 6$, where $r_{\pi}$ is the pion charge radius. 
We explicitly show all those properties of the off-shell pion form factors using the exactly solvable manifestly covariant model. The vertex function for the initial off-shell $\left(p^{2}=t\right)$ and final on-shell $\left(p^{\prime 2}=m_{\pi}^{2}\right) q \bar{q}$ bound-state pion coupled to the virtual photon with the 4-momentum $q$ in the fermion field theory can be calculated as

$$
\Gamma^{\mu}=i N_{c} g_{\pi q \bar{q}}^{2} \int \frac{d^{4} k}{(2 \pi)^{4}} \frac{\operatorname{Tr}\left[\gamma_{5}\left(\not k+\not q+m_{q}\right) \gamma^{\mu}\left(\not k+m_{q}\right) \gamma_{5}\left(\not k-\not k+m_{q}\right)\right]}{\left[k^{2}-m_{q}^{2}+i \varepsilon\right]\left[(k+q)^{2}-m_{q}^{2}+i \varepsilon\right]\left[(p-k)^{2}-m_{q}^{2}+i \varepsilon\right]},
$$

where $N_{c}$ is the number of colors and $g_{\pi q \bar{q}}$ corresponds to the coupling constant of the $\pi q \bar{q}$ vertex.

Using the Feynman parametrization and the dimensional regularization in $d(=4-2 \varepsilon)$-dimensions, we then obtain the two form factors $F_{1}\left(Q^{2}, t\right)$ and $F_{2}\left(Q^{2}, t\right)$ as

$$
\begin{aligned}
& F_{1}\left(Q^{2}, t\right)=-\frac{N_{c} g_{\pi q \bar{q}}^{2}}{8 \pi^{2}} \int_{0}^{1} d x \int_{0}^{x} d y\left[(1+3 y)\left(\gamma-\frac{1}{\varepsilon}+\frac{1}{2}+\log C\right)+\frac{\alpha}{C}\right], \\
& F_{2}\left(Q^{2}, t\right)=-\frac{N_{c} g_{\pi q \bar{q}}^{2}}{8 \pi^{2}} \int_{0}^{1} d x \int_{0}^{x} d y\left[3(1-2 x+y) \log C+\frac{2 \beta-\alpha}{C}\right]
\end{aligned}
$$

where $\gamma$ is the Euler-Mascheroni constant and $\alpha=(1+y)\left(E^{2}-m_{q}^{2}\right)-q \cdot E+2 y p \cdot E-y q \cdot p$, $\beta=(1-x+y)\left(E^{2}-m_{q}^{2}\right)+(1-2 x+2 y) p \cdot E+(x-y) q \cdot p$, and $C$ and $E$ are given in [5]. We should note that the form factor $F_{2}\left(Q^{2}, t\right)$ is free from the UV divergence since the integration of $(1-2 x+y)$ multiplied by the constant factor $(\gamma-1 / \varepsilon+1 / 2)$ gives zero in Eq. (2.4).

As the loop correction to the charge form factor $F_{1}\left(Q^{2}, t=m_{\pi}^{2}\right)$ must vanish at $Q^{2}=0$, the charge at $Q^{2}=0$ is given by a subtraction to the contribution by the loop integral. We thus redefine the renormalized charge form factor as

$$
F_{1}^{\mathrm{ren}}\left(Q^{2}, t\right)=1+\left[F_{1}\left(Q^{2}, t\right)-F_{1}\left(0, m_{\pi}^{2}\right)\right]
$$

where the loop correction in the square bracket vanishes at $Q^{2}=0$ and $t=m_{\pi}^{2}$ and the normalization of the electric charge is fixed by $F_{1}^{\text {ren. }}\left(0, t=m_{\pi}^{2}\right)=1$. The coupling $g_{\pi q \bar{q}}$ is related to the pseudoscalar coupling of the pion vis-á-vis partially conserved axial current. From the comparison of the pion decay constant $f_{\pi}$ using the same model, we obtain $g_{\pi q \bar{q}}=\left(2 m_{q} / f_{\pi}\right)$ by taking $F_{1}^{\text {ren }}\left(0, m_{\pi}^{2}\right)=1$. In our numerical calculation, however, we take $g_{\pi q \bar{q}}$ as another free parameter in addition to $m_{q}$ for the best fit of the model calculation compared to the experimental data. From now on, we shall denote $F_{1}^{\text {ren. }}\left(Q^{2}, t\right)$ as $F_{1}\left(Q^{2}, t\right)$ for convenience.

\section{Results}

In our numerical calculation, we tried to find the best fits of the form factor $F_{1}\left(Q^{2}, t\right)$ compared to the experimental data $F_{1}^{\text {Exp. }}\left(Q^{2}, t\right)$ for both the off-shell pion $\left(t \neq m_{\pi}^{2}\right)$ and the on-shell pion $\left(t=m_{\pi}^{2}\right)$ by adjusting our model parameters $\left(m_{q}, g_{\pi q \bar{q}}\right)$. We found the optimum ranges of quark masses, $0.12 \leq m_{q} \leq 0.16 \mathrm{GeV}$, and the best-fit for the coupling constants, $g_{\pi q \bar{q}}=(1.32,1.20,1.11)$ in unit of $\left(2 m_{q} / f_{\pi}^{\text {Exp. }}\right)$ for $m_{q}=(0.12,0.14,0.16) \mathrm{GeV}$, respectively. That is, our phenomenological best fit coupling constants $g_{\pi q \bar{q}}$ are not much different from the values of $2 m_{q} / f_{\pi}^{\text {Exp. }}$.

The overall landscape of the half-on-shell form factors, $F_{1}\left(Q^{2}, t\right)$ and $F_{2}\left(Q^{2}, t\right)$, obtained from $m_{q}=0.16 \mathrm{GeV}$ and $g_{\pi q \bar{q}}=1.11\left(2 m_{q} / f_{\pi}^{\text {Exp. }}\right)$ for spacelike regions are shown in Fig. 1 [5]. The 
figure represents the 3D plots of $F_{1}\left(Q^{2}, t\right)$ (top left), $-F_{2}\left(Q^{2}, t\right)$ (top right), $g\left(Q^{2}, t\right)$ (bottom left) and the sum rule (bottom right) given by Eq. (2.2) for the momentum transfer region $0 \leq Q^{2} \leq 3$ $\mathrm{GeV}^{2}$ and $m_{\pi}^{2} \geq t \geq-0.4 \mathrm{GeV}^{2}$. While the form factor $F_{2}\left(Q^{2}, t\right)$ goes to zero as $t \rightarrow m_{\pi}^{2}$, the form factor $g\left(Q^{2}, t\right)$ is nonzero even in the on-mass-shell limit. Furthermore, $F_{1}(0, t)$ shows some dependency on $t$, which is necessary to know in the case of extracting $F_{2}\left(Q^{2}, t\right)$ from the pion electroproduction data.
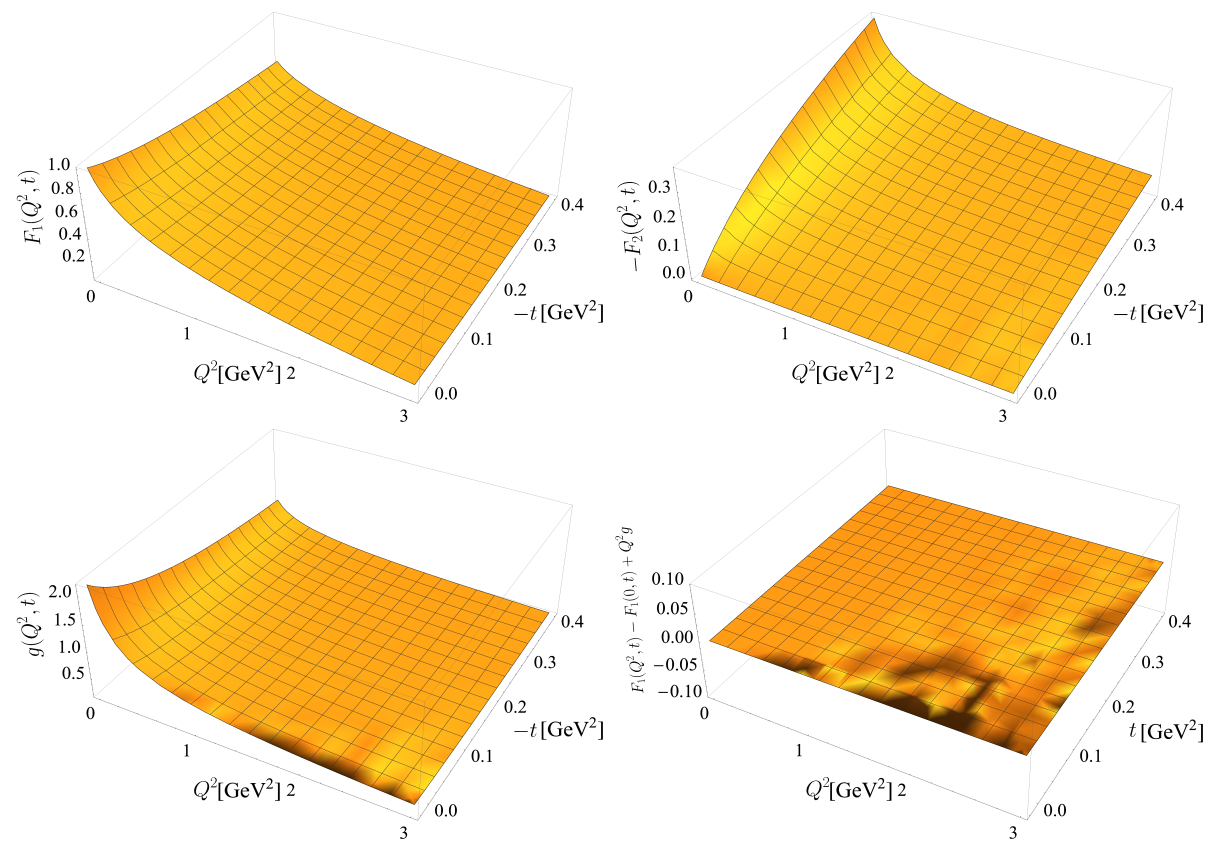

Figure 1: The 3D plots of $F_{1}\left(Q^{2}, t\right)$ (top left), $F_{2}\left(Q^{2}, t\right)$ (top right), $g\left(Q^{2}, t\right)$ (bottom left) and the sum rule (bottom right) given by Eq. (2.2) for the spacelike momentum transfer region $0 \leq Q^{2} \leq 3 \mathrm{GeV}^{2}$ and $m_{\pi}^{2} \geq t \geq-0.4 \mathrm{GeV}^{2}$.

The on-shell pion form factors $F_{1}\left(Q^{2}, m_{\pi}^{2}\right)$ (black lines) and $g\left(Q^{2}, m_{\pi}^{2}\right)$ (blue lines) from the covariant model for the spacelike region $Q^{2}>0$ are shown in Fig. 2 and compared with the extracted values of $F_{1}^{\text {Exp. }}\left(Q^{2}, t=m_{\pi}^{2}\right)$ (black data) and $g^{\text {Exp. }}\left(Q^{2}, t=m_{\pi}^{2}\right)=\left[1-F_{1}^{\text {Exp. }}\left(Q^{2}, t=m_{\pi}^{2}\right)\right] / Q^{2}$ (blue data) [5]. The model parameters in Fig. 2 are $m_{q}=(0.12,0.16) \mathrm{GeV}$ using the variation of the couplings $g_{\pi q \bar{q}}=(1.32 \pm 0.04,1.11 \pm 0.04)\left(2 m_{q} / f_{\pi}^{\text {Exp. }}\right)$, respectively. The solid and dashed lines represent the results obtained from $m_{q}=0.12$ and $0.16 \mathrm{GeV}$ using the upper and lower limits of the corresponding $g_{\pi q \bar{q}}$. Unlike the form factor $F_{2}\left(Q^{2}, t\right)$, the form factor $g\left(Q^{2}, t\right)$ does not vanish in the on-shell limit. We note that the current Particle Data Group [6] average $r_{\pi}^{\operatorname{Exp}}=\sqrt{\left\langle r_{\pi}^{2}\right\rangle}=(0.672 \pm 0.008) \mathrm{fm}$ for the rms value of the pion charge radius corresponds to $g^{\text {Exp. }}\left(Q^{2}=0, m_{\pi}^{2}\right)=(1.953 \pm 0.023) \mathrm{GeV}^{-2}$.

\section{Conclusions}

We investigated the pion electromagnetic half-off-shell form factors $F_{1}\left(Q^{2}, t\right)$ and $F_{2}\left(Q^{2}, t\right)$ using the manifestly covariant fermion field theory model. We also note that the ratio of $F_{2}\left(Q^{2}, t\right)$ 


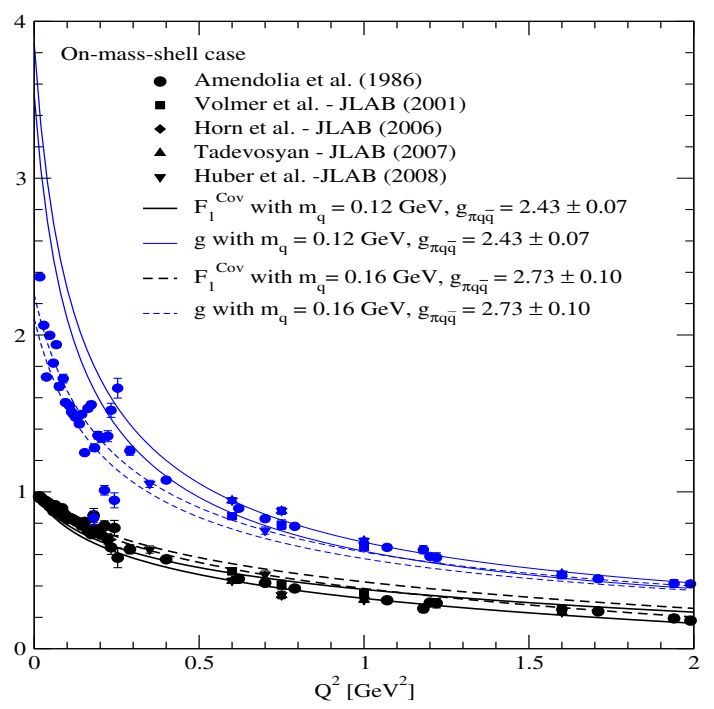

Figure 2: The on-shell pion form factors $F_{1}\left(Q^{2}, m_{\pi}^{2}\right)$ (black lines) and $g\left(Q^{2}, m_{\pi}^{2}\right)$ (blue lines) for the spacelike momentum transfer region $0 \leq Q^{2} \leq 2 \mathrm{GeV}^{2}$ compared with the experimental data $[1,2,3,7]$ for $F_{1}^{\text {Exp. }}$ (black data) and $g^{\text {Exp. }}$ (blue data).

to $t-m_{\pi}^{2}$ is nonzero in the limit of $t \rightarrow m_{\pi}^{2}$ while $F_{2}\left(Q^{2}, t\right)$ goes to zero as $t \rightarrow m_{\pi}^{2}$. This led us to define the new form factor $g\left(Q^{2}, t\right)=F_{2}\left(Q^{2}, t\right) /\left(t-m_{\pi}^{2}\right)$, which should be measurable even in the on-mass-shell limit on par with the usual charge form factor $F_{1}\left(Q^{2}, m_{\pi}^{2}\right)$. In particular, we obtain the sum rule which relates $g\left(Q^{2}, t\right)$ to $F_{1}\left(Q^{2}, t\right)$ and note that the value of $g\left(Q^{2}=0, t=m_{\pi}^{2}\right)$ corresponds to the charge radius of a pion. According to Eq. (2.2), however, one needs the information of $F_{1}(0, t)$ to determine $g\left(Q^{2}, t\right)$, while no data of $F_{1}\left(Q^{2}, t\right)$ exist at $Q^{2}=0$ for $t<0$.

In this work, we used a simple covariant model to provide at least a clear example of demonstration for the simultaneous extraction of both $F_{1}\left(Q^{2}, t\right)$ and $g\left(Q^{2}, t\right)\left(\right.$ or $\left.F_{2}\left(Q^{2}, t\right)\right)$. In our numerical calculations, we show the 3D plots of $F_{1(2)}\left(Q^{2}, t\right)$ and $g\left(Q^{2}, t\right)$ in terms of $\left(Q^{2}, t\right)$ values as shown in Fig. 1. It encourages more in-depth theoretical and experimental efforts to reveal the 3D imaging of the off-shell pion form factors.

\section{References}

[1] H. P. Blok et al., Phys. Rev. C 78, 045202 (2008); G. M. Huber et al., Phys. Rev. C 78, 045203 (2008).

[2] T. Horn et al., Phys. Rev. Lett. 97, 192001 (2006); Phys. Rev. C 78, 058201 (2008).

[3] J. Volmer et al., Phys. Rev. Lett. 86, 1713 (2001); V. Tadevosyan et al., Phys. Rev. C 75, 055205 (2007).

[4] T. E. Rudy, H. W. Fearing, and S. Scherer, Phys. Rev. C 50, 447 (1994).

[5] H.-M. Choi, T. Frederico, C.-R. Ji, and J.P.B.C. de Melo, Phys. Rev. D 100, 116020 (2019).

[6] M. Tanabashi et al. (Particle Data Group), Phys. Rev. D 98, 030001 (2018).

[7] S. R. Amendolia et al., Nucl. Phys. B 277, 168 (1986). 